\title{
Carbon Monoxide Monitoring System Based On Arduino-GSM for Environmental Monitoring Application
}

\author{
Ms. Nishigandha Kaneri ${ }^{1}$, Ms. Kajal Bhalekar ${ }^{2}$, Ms. Sajari Kharvatkar ${ }^{3}$ \\ ${ }^{1,2,3}$ Electronics \& Telecommunication, VPM's MPCOE Velneshwar \\ Email:nishi.kaneri@gmail.com, kajalbhalekar8@gmail.com, sajarikharvatkar8@gmail.com
}

\begin{abstract}
In recent years with tremendous progress in technology and growth in demand for vehicles, an individual fizzle to look after awful factors that occur due to improper maintenance of vehicles results in increase in air pollution and which results in disturbing environmental condition all over the world, that tends to raise global warming. In this paper "Carbon Monoxide Monitoring System Based on Arduino-GSM for Environmental Monitoring Application" is a system which detects the Carbon Monoxide (CO) gas coming out of a vehicle which is displayed on vehicle itself, so when the level of $\mathrm{CO}$ increases alerts will be sent to the owner of vehicle regarding maintenance of vehicle and if action is not taken regarding $\mathrm{CO}$ the vehicle is made to stop and later allowed to start the vehicle after given period. This cycle is continued to keep the CO free environment and also reduce human efforts to monitor each and every vehicle. The repetitively breaking down of a vehicle causes the owner to take serious action for reducing the $\mathrm{CO}$ of his vehicle.

Index Terms: Carbon Monoxide, sensor module, Arduino \& GSM module.
\end{abstract}

\section{INTRODUCTION}

Carbon Monoxide Monitoring System Based on Arduino-GSM for Environmental Monitoring Application system that totally minimizes the hazardous gas that prolonged exposure through different vehicles has causing the ozone layer depletion causing skin cancer, global warming, stress, headache, dizziness, asthma problem and also it decreases the life of a vehicle.Therefore the need of monitoring air pollution around the city and the public transport and private vehicles is a very important issue today [1]. CO is exhausted from vehicle's, furnace, oven, stove, fireplace, water heater, portable generator, charcoal grill but a major source of $\mathrm{CO}$ is vehicle's exhaust due to incomplete combustion of carbon-containing fuels [4]. This instrument reduces manpower and also increases the overall flexibility, compatible with any petrol vehicle and has the ability to work independently.

The majority of the population in the world do prefer vehicles for even short distance for travelling but no one actually cares about what would be the adverse effects of $\mathrm{CO}$ if it is exhausted in a large amount and not taking the serious measures of the same so this instrument will help to overcome the effects of CO.

The sensor is used to detects the amount of $\mathrm{CO}$ that has been exhausted from the vehicle's silencer. The CO here is measured in the form of PPM (Parts per Million), in percentage as well as it shows the levels (low, medium or high) and this is displayed respectively.
The PPM is calculated, the levels are displayed. If the level goes low, medium or even high the same is displayed and then that is to be acknowledged to the owner of the vehicle so, after crossing the high level an alert/warning is given in the SMS form to an owner of a vehicle. These alert messages will be for taking serious measures to reduce the $\mathrm{CO}$ factors so the driver doesn't come up with ending up stopping his/her vehicle all sudden. There will be total 3 alerts given with a particular interval of time. The last warning will be of only to inform the owner that within how much time span the vehicle will stop so that even he/she is well known for the action that is going to be taken. Now after restricting the vehicle for a certain period of time, to start again the vehicle we are giving another chance to the owner for maintaining the vehicle's $\mathrm{CO}$ level so we allow them to restart the vehicle through this instrument only.

These above steps will keep on continue so that on an average the $\mathrm{CO}$ level is taken into consideration for strictly reducing the level of $\mathrm{CO}$ to make our city as well country pollution free.

\section{PROBLEM STATEMENT}

An individual adopts vehicle daily but with its ratio of usage, it is also individuals responsibility to take care of the pollution that is been increasing. People hardly make sure to service their vehicle or take care of the $\mathrm{CO}$ level. As vehicles are important in day to day life and even the hazardous gas are also to be minimized and balanced the environmental condition. So our instrument is solution for all these problems. 


\section{OBJECTIVE}

The level of $\mathrm{CO}$ is measured and displayed on LCD for the owner/driver acknowledgment. The monitoring is done on the daily basis that is when it exceeds particular level it will keep track on the messages it has given till the date to the owner. This system plays a vital role to increase the life of the vehicle and making an individual responsible for the global warming.

\section{LITERATURE SURVEY}

Suganya et al investigated Wireless Sensor Network (WSN) that keeps track on a number of cars, buses, motorbikes etc in the city. This plays an important role in the application of environmental monitoring [1]. Where the communication, as well as monitoring, becomes wireless. This research concentrates in the gas level in the air. Its main objective is to monitor NO2, Humidity, Temperature \& $\mathrm{CO}$ in the air [1]. MANET (Mobile Ad Hoc Network) routing algorithm is used in their invention

S.Suriya Jothi et al invented that Air pollution is one of the major concerns that affects human health and life of plants and animals [6]. For avoiding such circumstances, the root causes should be closely monitored. Hence vehicle is a very important factor for producing $\mathrm{CO}$ at a large amount. So the commercial sensor MQ7 is used to measure the $\mathrm{CO}$ and display in the graphical form using Java GUI. The software part of the project is developed under JAVA net beans IDE interfaced [6].

Khunarak $\mathrm{C}$ et al [4] forced on the development of real-time solution [4]. Use of ZigBee, sensor nodes, GPS to construct distributed system for urban air pollution monitoring and control. ZigBee module and pollution server are interfaced with GPS system to display real-time pollutants levels and their location on a $24 \mathrm{~h} / 7$ days basis. In this system, they have established 4 nodes which will indicate 4 harmful gases such as $\mathrm{CO} 2, \mathrm{SO} 2 \& \mathrm{NO} 2$ etc.

Moshin Murad et al proposed a system where the prevention of $\mathrm{CO}$ based is on a Wireless Sensor Network (WSN) system, which continuously detects the $\mathrm{CO}$ level in a restrained space like in steel mills and upon alarming conditions, automatically activates countermeasure system to lower the $\mathrm{CO}$ concentration level [3]. His research also presents the implementation of a WSN monitoring system connected to a customized actuator circuit which can switch the exhaust and alarm systems on and off autonomously [3].

\section{FLOW CHART}

The flow chart is an elementary method to understand the concept of the system. This flow chart explains the detection and monitoring of the $\mathrm{CO}$.

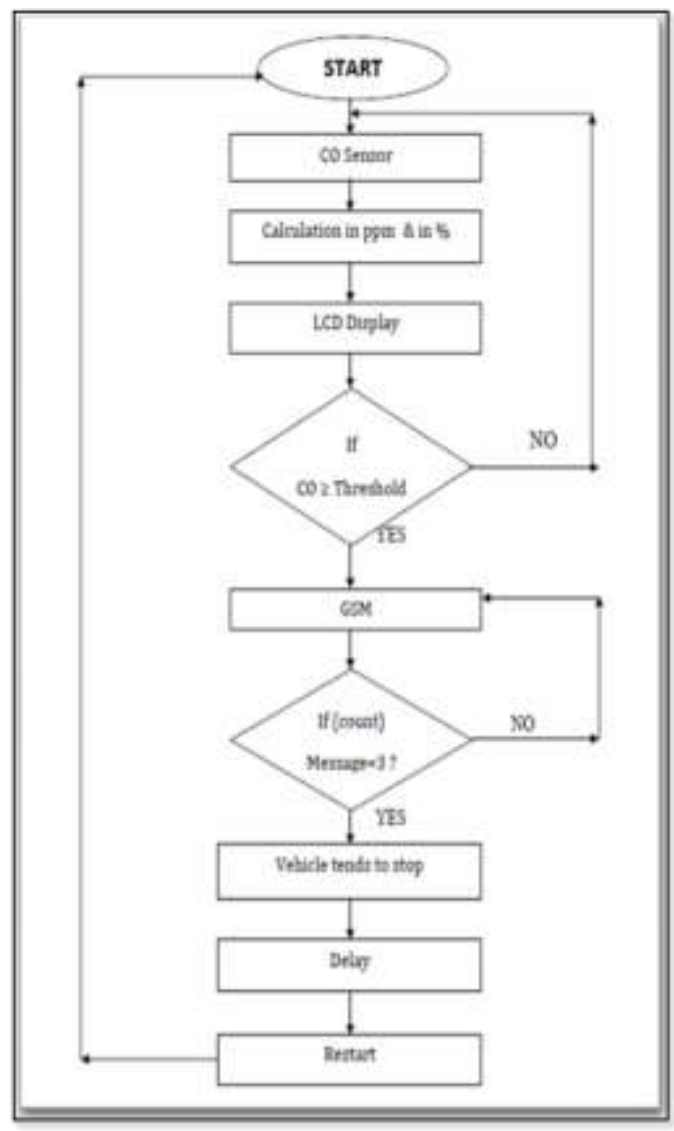

Fig 1: Flow chart

\section{METHODOLOGY}

A] Procedural Method of Study:

\section{1] CO Sensor:}

The MQ7 is semiconductor CO sensor, it is sensitive to combustible gas which is highly formed from the vehicle, sensor used in the system detects the amount of CO gas from the vehicle and hence similarly readings are obtained as in analog voltages form. Its concentration is from 10-10000ppm CO. The operating voltage is $5 \mathrm{~V} \mathrm{DC}$. The sensor operates within the temperatures range -20 to $50^{\circ} \mathrm{C}$ and consumes less than $150 \mathrm{~mA}$ at $5 \mathrm{~V}$. The sensitivity characteristic of the MQ7 sensor depends on the ratio (Rs/Ro) of the Sensor Resistance (Rs), when sensor is exposed to $\mathrm{CO}$ gas then its initial sensor Resistor tends to vary (R0) i.e. of $10 \mathrm{~K} \mathrm{Ohm}$. The analog output voltage is now calculated to get the value in PPM form and in percentage. The value retrieved from this is derived from the formula using $\mathrm{C}$ language on Arduino platform. Figure 1 shows the graphical representation of MQ7 $\mathrm{CO}$ matching the result. 


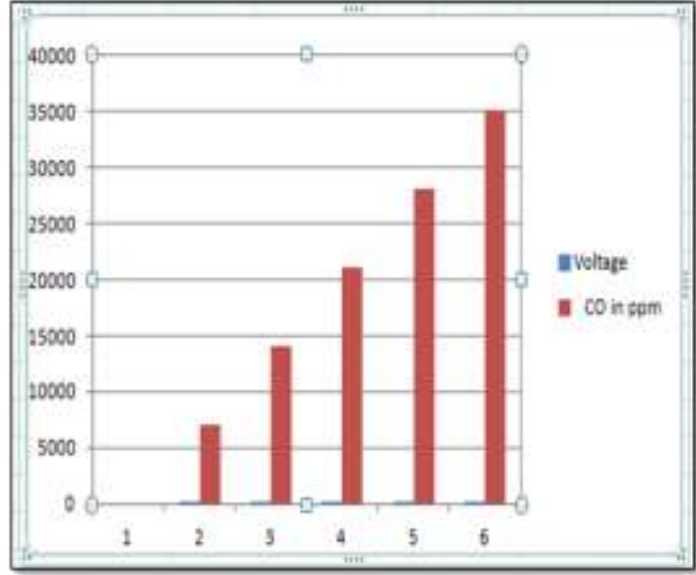

Fig 2. Graph of voltage v/s CO in ppm

\section{2] SIM-900 GSM Module:}

In this instrument, SIM-900 GSM Module is used to send the message after it is been acknowledged by the program. GSM (Global System for Mobile Communication) is an open digital cellular based technology used for transmitting mobile voice or data services. In this instrument, we have used to send the alert using GSM Module.

GSM is mainly classified into two part, GSM 900 \& GSM 1800 which is used in most part of the world. The watts are limited for handset to a maximum of 1 watt for GSM $(1800 / 1900 \mathrm{MHz})$ and where as 2 watts GSM $(850 / 900 / 300 \mathrm{MHz})$ respectively.This is fully compatible with Arduino, as the request is made from the Arduino it will send the alert message to that particular vehicle's owner.

\section{B] BLOCK DIAGRAM:}

The block diagram simplifies the outcome of each step. The initial stage is the sensor which calculates the $\mathrm{CO}$ and displays on LCD and the increased amount of $\mathrm{CO}$ will be made to acknowledge the customer from GSM module. This whole system will be installed in the vehicle itself with sealed mechanism so that environmental changes do not affect the smooth working of the system.

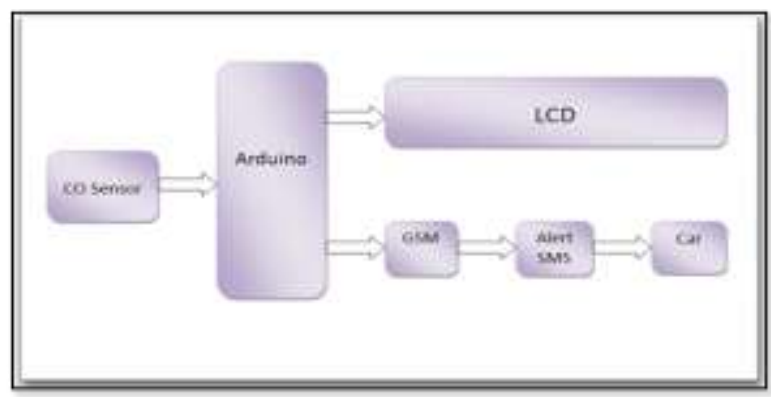

Fig 3: Block Diagram of the system

\section{C] RESULTS}

A) Output of Sensor:
The sensor gives 3 phenomenal conditions that are the change in the level on $\mathrm{CO}$ based of calculation and calibration:
i. Low level
ii. Medium level
iii. High level

1) Low Level: Initially the level of $\mathrm{CO}$ will be low as you start your vehicle so, the $\mathrm{CO}$ is displayed under limit and accordingly the display will show the readings. If the vehicle is well maintained and serviced periodically then display will continue showing as "Low level" and the owner will not receive any message regarding the same.

2) Medium level: The $\mathrm{CO}$ reading shows medium level when it crosses the threshold which has been initialized in the formula and same will be displayed and respectively no message will be given to the owner.

3) High Level: This is the actual level when action is takes place i.e. the GSM now will send the warning message to the owner of the vehicle.

Depending upon all these above cases the whole mechanism is going to work.

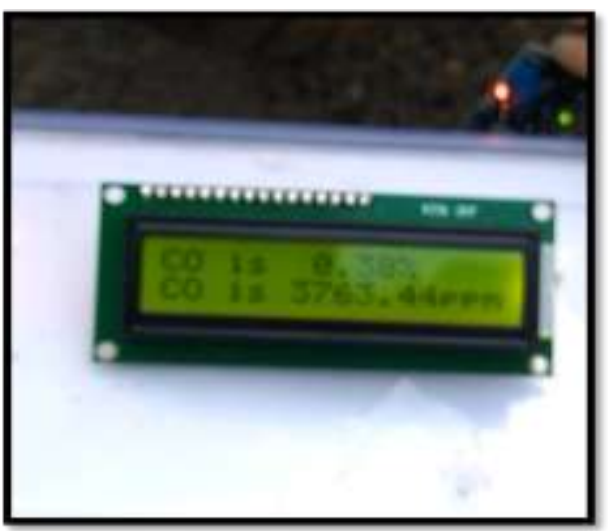

Fig 4: Result on LCD

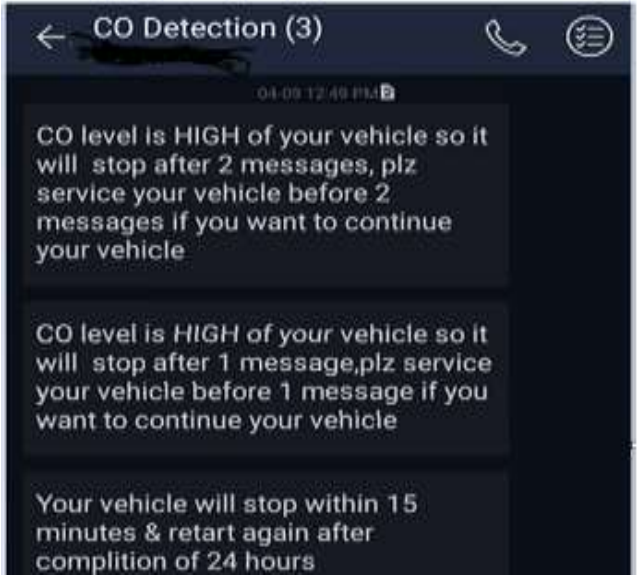

Fig: 5 Alert in SMS form (screenshot) 
This is the alerts that are given to the owner of the vehicle. There are total 3 alerts .each is given on daily bases as shown in above fig 5 (screenshot). The time delay for stopping the vehicle is given 15 minutes we can increase the time delay, to make sure that user is not in trouble, sufficient time could be decided by the concerned authority.

\section{B) Verified Result:}

We visited REGINOL TRANSPORT OFFICE, RATNAGIRI and demonstrated the project in front of concern authority. As the main aim of our project is to reduce $\mathrm{CO}$, the RTO officer found it useful for vehicles. The CERTIFICATION given by the RTO is attached further.

\section{CONCLUSION}

$\mathrm{CO}$ Detection and Monitoring system are developed using ATmega - Arduino C language and interfacing MQ7 sensor module for sensing the CO level and GSM for SMS and LCD for display. This instrument is successfully implemented and tested on motorbike, as the bike started the $\mathrm{CO}$ sensor showed the level in percentage and ppm and according with the variation that took places the display shows the reading and alert in the form of SMS was given as the $\mathrm{CO}$ level increased and after 3 warning, owner regarding servicing of the vehicle, even after that alerts the precautions were not taken so the vehicle stopped and did not start until the interval of punishment and after a particular period of time the bike was allowed to start in normal mode. Again after this if the CO level is not maintained by the owner this cycle will keep on continue. This continuation will make sure that pure petrol is used in vehicle to overcome such alerts and one who is supplying 'petrol mixed' fuels can be caught easily which is 'Biggest scam of India' and banned further to stop this crime to take place. This serious action taking mechanism doesn't include any $3^{\text {rd }}$ party; the whole system is between the owner and automated system that is installed in the vehicles.

\section{FUTURE SCOPE}

The system is now totally wired mechanism with good reliability and fast response for controlling the large amount of CO. Further advancement may include wireless. That means we may go for Bluetooth that is, the connection from sensor to Arduino will be made connection free so that the wiring cost and maintenance are totally eliminated.

The messaging process can also be made advance by giving alert to the 'Whatsapp' phone application number. The Cloud computing concept for the database can also be introduced for the purpose of storing data.

\section{REFERENCES}

[1]. Suganya E, Vijayashaarathi S "Smart Vehicle Monitoring System for Air Pollution Detection using Wsn" International Conference on Communication and Signal Processing, April 6-8, 2016 IEEE, India

[2]. Abdul Hadi Nograles H , Agbay, Christopher Paolo D "Low Cost Internet Based Wireless Sensor Network for Air Pollution Monitoring using Zigbee Module" ISBN: 978-1-4799-3724-0/14/\$31.00 @2014 IEEE

[3]. Mohsin Murad, K. M. Yahya, G. M. Hassan, Muhammad Fahim Jan, Qaiser Habib, Muhammad Irfan "Carbon Monoxide Detection and Autonomous Countermeasure System for a Steel Mill using Wireless Sensor and Actuator Network" 2010 6th International Conference on Emerging Technologies (ICET)

[4]. Khunarak, C., Lutz, M., Kerdcharoen, T. "Wi-Fi Electronic Nose For Indoor Air Monitoring" IEEE Conference, pp. 1-4, (May 2012).

[5]. A. R. Al-Ali,, Imran Zualkernan, and Fadi Aloul, "A Mobile GPRS-Sensors Array for Air Pollution Monitoring", IEEE Sensor Journal, Vol. 10, No. 10, (October 2011)

[6]. S.Suriya Jothi, Y.Jeyasingh "System To Monitor And Prevent The Air Pollution By Reducing Co Emission" International Journal Of Computing Communication And Information System (IJCCIS) Vol.8. No.1 2016, Pp. 21-27@Gopalax Journals, Singapore

[7]. N. Kularatna and B. H. Sudantha, "An environmental air pollution monitoring system based on the IEEE 1451 standard for low cost requirements," IEEE Sensors J., vol. 8, pp. 415-422, Apr. 2008.

[8]. Alave, K., (2010). DENR Issues New Limits on Vehicular Emissions to Cut Air Pollution. Philippine Daily Inquirer, pages 12-13.

[9]. Haibing Hu, Gang Wang, Qixing Zhang, Jinjun Wang, Jun Fang, Yongming Zhang, "Design Wireless Multi-sensor Fire Detection and Alarm System Based on ARM", In proceedings of The Ninth International Conference on Electronic Measurement \& Instruments, ICEMI'2009

[10]. DOSH. Industry Code of Practice on Indoor Air Quality; Department of Occupational Safety and Health (DOSH): Kuala Lumpur, Malaysia, 2010.

[11]. Burgonio, T. (2007). Philippine DailyInquirer, Air pollution kills nearly 5,000 Metro residents yearly. Pages 5 6Retrieved from http://newsinfo.inquirer.net 
Miss. Nishigandha Kaneri.et.al. Int. Journal of Engineering Research and Application www.ijera.com ISSN : 2248-9622, Vol. 7, Issue 5, ( Part-2) May 2017, pp.57-61

\section{CERTIFICATE:}

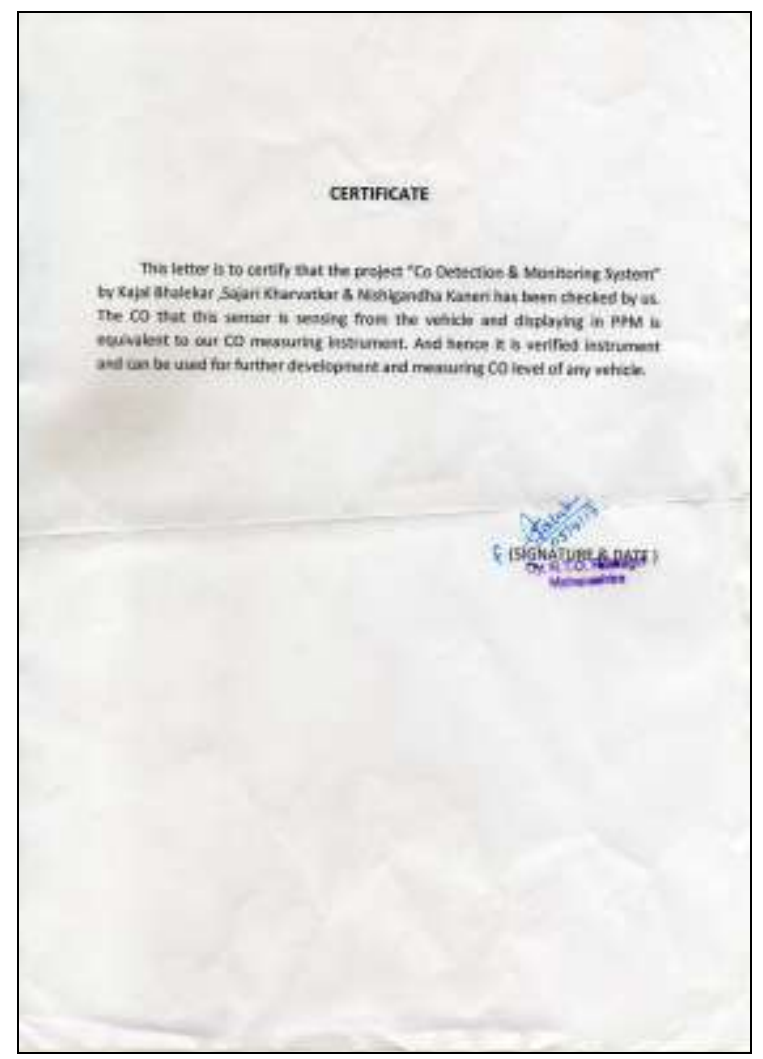

